\title{
Present Status and Trend of Development of Transmission Fluid Simulation Software
}

\author{
Hongmei Liu ${ }^{1, a^{*}}$, Le $\mathrm{Mi}^{2, \mathrm{~b}}$, Yi Lv ${ }^{2, \mathrm{c}}$ and Jianjun Wang ${ }^{2, \mathrm{~d}}$ \\ ${ }^{1}$ Engineering Training Center, Nantong University, Nantong, Jiangsu 226019, China \\ ${ }^{2}$ School of Mechanical Engineering, Nantong University, Nantong, Jiangsu 226019, China \\ a*liu.hm@ntu.edu.cn ,b1650055720@qq.com ,"260443169@qq.com ,d851532953@qq.com
}

Keywords: fluid drive; computer simulation; CFD

Abstract. The developmental history and progress of the fluid transmission are introduced in this paper. As well as the importance of the simulation software about fluid drive is pointed out, the advantages and disadvantages of several typical fluid driving simulation software (CFD) between domestic and international are comprehensively analyzed. Finally, the development trend of fluid transmission simulation is introduced.

\section{Introduction}

CFD software is short for Computational Fluid Dynamics. It is modern fluid mechanics, the numerical mathematics and computer science combined with the product, and is an edge of the strong vitality of science. It was based on the electronic computer as the tool, application of various mathematical method of discretization, the convection strength of all kinds of problems in the numerical experiment, computer simulation and analysis, in order to solve various practical problems ${ }^{[1]}$. It is integrated with the academic disciplines, has the remarkable characteristics of mechanical and electrical integration of liquid, especially combined with computer technology, makes the hydraulic technology in the system design, control, fault diagnosis, virtual reality and so on have made considerable progress. In 1726, Bernoulli is starting from the classical mechanics of conservation of energy, study the movement of water through the water supply pipe, test analysis, obtained the fluid under the constant movement of the relationship between the flow velocity, pressure, flow channel height Bernoulli equation ${ }^{[2]}$. The establishment of the Euler equation and Bernoulli's equation, is a fluid dynamics as a symbol of a branch to build, began to use differential equation and experimental measurement of fluid motion quantitative research stage. A central computer access to all components of the current features, and compared with the standard features, if significantly beyond, may issue a warning that a necessary. With the continuous development of the central computer software, such as logic judgment, spectrum analysis and wavelet analysis, etc., can further realize the fault diagnosis of hydraulic system.

\section{Principle of Fluid Analysis Software}

Computational fluid dynamics and related calculation of heat transfer, the principle of combustion science is calculated by using numerical methods to solve nonlinear simultaneous mass, momentum, energy, components and custom scalar differential equations, from solving the results can predict flow, heat and mass transfer, combustion and other process details and become a powerful tool for process optimization and amplification means quantitative design. The basic characteristics of computational fluid dynamics are numerical simulation and computer experiment, it embarks from the basic physical theorem, and replaces the expensive laboratory equipment of fluid dynamics in a large part ${ }^{[3]}$. It has a significant impact on the scientific research and engineering technology and has been a powerful international research field. The computational fluid dynamics are the core and important technology to conduct heat transfer, mass transfer, momentum transfer, multi-phase flow, and chemical reaction. It has been widely used in aerospace design, car design, biomedical engineer, chemical processing, design of turbine, semiconductor design, HAVC \& R (Heating, Ventilation and Air Conditioning) and many other engineering fields ${ }^{[4]}$.

CFD software in general consist of Pre-Processing, solver, Post-Processing ${ }^{[5]}$. Each part has its 
unique function, respectively, just as shown in Table. 1.

Table. 1 Parts of CFD

\begin{tabular}{|c|c|c|c|}
\hline & Pre- Processing & Solver & Post-Processing \\
\hline Function & $\begin{array}{l}\text { Geometry Model } \\
\text { Network Partition }\end{array}$ & $\begin{array}{l}\text { Determine the control equation of CFD method } \\
\text { Select discrete method to discrete } \\
\text { Selection of numerical calculation method } \\
\text { Input related parameters }\end{array}$ & $\begin{array}{l}\text { Velocity field, temperature field, pressure field } \\
\text { and other parameters of computer visualization } \\
\text { and parameterized treatment. }\end{array}$ \\
\hline
\end{tabular}

\section{Development of CFD Software}

\section{CFX}

CFX is developed by the AEA British Company, and is a kind of practical fluid engineering analysis tool. It is used to simulate the fluid flow, heat transfer, multiphase flow, chemical reaction, burning questions. Its advantage lies in current physical phenomena in simple but complex geometry problems. It applies to the rectangular/cylindrical/rotating coordinate system, steady/unsteady flow, the transient/sliding grid, incompressible, weakly compressible, compressible fluid, buoyancy flow, multiphase flow, non-Newtonian fluid, chemical reaction, burning, $\mathrm{NO}_{\mathrm{x}}$ formation, radiation, and mixed heat transfer process of porous media. CFX uses the limited element method, the automatic time step control, SIMPLE algorithm, algebraic multigrain, ICCG, Line Stone, Stone and Block method $^{[6]}$, so that it can effectively and accurately express the complex geometry, connection module and construct any desired geometry. Within each module, the generation of grid can be rapid and effective, for example, the calculation of the movement of the cylinder piston and the movement of free surface allow more expansion and deformation. Sliding grid allows all parts of the grid can slip or rotate relative, this function can be used to calculate fluid interaction between cone bit and the wall. CFX introduced various recognized the turbulence model. For example: k - e model, low Reynolds number k - e model, RNG, algebraic Reynolds stress model, the differential Reynolds stress model, differential Renault flux model, etc. The CFX multiphase flow model can be used for various flow analyses in industrial production, including the single particle motion model, continuous phase and disperse phase model of multiphase flow and free surface flow model.

\section{FLUENT}

FLUENT software is relatively popular in the international commercial CFD software package, the market share of $60 \%$ in the United States. Whenever with the relevant industrial fluids, heat transfer and chemical reactions can be used. It has the rich physical model, the advanced numerical methods a strong Pre-Processor and Post-Processor functions, in the aerospace, automobile design, oil and gas, turbine design has been widely used in such aspects as ${ }^{[7]}$.

Fluent software design is based on the idea of CFD software group, from the perspective of user needs, according to various complex physical phenomena, Fluent software using numerical methods and different discrete format, in a specific field in order to make computing speed, stability and accuracy to achieve the best combination, so as to efficiently solve the complex flow calculation problems in various fields. Developed based on the above ideas, Fluent flow simulation software, applicable to all areas of the software to simulate fluid flow, heat and mass transfer, chemical reaction and other complex physical phenomena.

\section{FloEFD}

FloEFD is a new branch of CFD- - synchronization of CFD application products. It is a functional general CFD tool which is able to system integrating with Catia, NX and Creo ${ }^{[8]}$. FloEFD has a simple and automatic Pre-Processing to mesh generation. The process of handling simulation, including 3D CAD model analysis, mesh generation, visualize solving and result, are contained within a package. 
FloEFD is based on the same mathematical principles with traditional CFD software, but it distinguishes itself with seven key technologies to be more simple and powerful. Combine with your MCAD software, FloEFD can bring infinite ability on the simulation. It can improve product performance, functionality and reliability, reduce the physical prototype and production costs, and minimize the risk of design errors. Computational fluid dynamics (CFD) is a breakthrough technology. With the help of CFD, design engineers can perform the front-end and synchronization of analysis throughout the product life cycle in their familiar MCAD interface.

Compared with the traditional CFD tools, synchronous CFD can shorten as high as $65-75 \%$ of the time, convenient for the user to optimize product performance and reliability, reduce the experimental samples and research and development costs, eliminate losses caused by the date of delivery or product quality problems.

\section{PHOENICS}

Phonics is a general CFD software which was developed by the CHAM British Company to simulate the heat transfer, flow, reaction, the combustion process, it has a history of more than 30 years. Grid system includes the vertical Angle, columns, curved surface (including non orthogonal grids and sports, but in the VR environment can't), multiple grids, precise grid.

It can simulate the three dimensional steady or unsteady compressible flow or incompressible flow, including non Newtonian flow, flow in porous media, and can consider the effect of viscosity, density, temperature changes. On fluid model, phonics were built-in 22 kinds of turbulence model which are suitable for all kinds of Re number of occasions, including turbulent Reynolds stress model, fluid model, the flux model, and various variations of $\mathrm{k}$ - e model, a total of 21 turbulence models, eight multiphase flow models, more than 10 different schemes. Phoenics VR (virtual reality) color in the graphical interface menu system is the most convenient for the Pre-Processing in this a few CFD software. It can be directly read in the Pro / E model (needed to convert the STL format), make complex geometry, generate more convenient, in the definition of the boundary conditions is also very simple, and the grid automatically generated. But the downside is that the grid is a coarse, for complex curved surface or small curvature of the grid, it cannot be subdivided, which is said that it cannot use a grid in VR environments. On the other hand, VR Post-Processing of also is not very good. To undertake more advanced analysis command format is used, but it will be sent on the ease of use than other software.

In addition, Phoenics comes with more than 1000 examples and validation, accompanied by complete input file which can be read and changed, for example, CHAM company do a flow field analysis of PDC bit. Software Phoenics' openness is very good, for the existing model is modified, adding new model function and interface, can be a secondary development in the FORTRAN language.

\section{STAR-CD}

STAR-CD is short for Simulation of Turbulent flow in Arbitrary Region, CD is short for Computational Dynamics Ltd. It is a general fluid calculation software based on the finite volume method, in terms of grid generation, using unstructured grids, available for hexahedral element, tetrahedron, triangular prism of the interface, cone and six in the shape of a pyramid polyhedron, still can interface with CAD, CAE software, such as ANSYS, IDEAS, NASTRAN, PATRAN, ICEMCFD, GRIDGEN etc. It can adapt to the complex STAR - CD in areas of special advantage.

STAR - $\mathrm{CD}^{[9]}$ can handle mobile grid, used for the calculation of the multi-stage turbine. In terms of different format, it brings into the first-order UpWIND, the second order UpWIND, CDS, QUICK, and the first-order UpWIND mixed with CDS or QUICK format. In the aspect of pressure coupling, it uses SIMPLE, PISO, and an algorithm called SIMPLO. In terms of the turbulence model, using the k-e model, RNK-ke model, ke two layer model, it can compute many problems about the steady state and transient, Newton, non-Newtonian fluid, porous media, subsonic, supersonic, multi-phase flow. 


\section{Development direction of CFD Software}

Firstly, system modeling is not easy. The primary task for the simulation of hydraulic system is to establish mathematical model, the most difficult work is modeling, and then may be a simulation, while the modeling is a very complicated work. The model is directly related to the emulation results, inappropriate model is possible to derive the opposite result, but also the builder may not know. It may result in huge loss ${ }^{[10]}$. Presently, the vast majority of software uses the equation of state modeling, but also some software uses the transfer function or bond graph modeling. It is difficult for the general hydraulic workers to use the transfer function modeling, especially the bond graph modeling.

Secondly, the accuracy and reliability of the system is not high. To make the system simulation technology realm, we have to improve the accuracy and reliability of the simulation results. Under the conditions allowed by the system, precision is higher, the simulation result is more reliable and accurate, but because of the hydraulic simulation software itself and simulation technology and other aspects, the accuracy of the simulation results can't be very high ${ }^{[11]}$. The principle or method of modeling, model simplification, incorrect selection of original date of model deviation and computer performance, those, which have not been resolved today, will reduce the accuracy of simulation results, and may affect the development and application of simulation technology.

Finally, the distributed interactive simulation and real-time simulation technology, the object-oriented simulation technology and carrying out a research into the man-machine harmonious environment simulation, artificial intelligence and expert system simulation environment harmonious human-computer simulation technology in the application of semi - physical simulation technology or the development direction of CFD software. To solve the problem of subway station traffic organization, for example, social force by CFD software simulation and imitation fluid mechanics simulation, the imitation fluid mechanics model is relatively closer to the real environment of people, effectively relieve the traffic congestion problem of disorder.

\section{Acknowledgments}

This work is supported by Application Research Project of Jiangsu Economic and Information Technology Commission ([2013]742-1).

\section{References}

[1] Yong-Liang Wang, Yin Lu, Zhen-Peng Zhao. The present situation and development trend of hydraulic simulation software [J]. Chinese Hydraulics and Pneumatics, 2012,08:1-4. (in Chinese)

[2] Yi-Qun Wang,Wei-Zhang. Fluid drive and control technology are reviewed [J]. Chinese Journal of Mechanical Engineering, 2003,10:95-99. (in Chinese)

[3] Xi-Shu Deng, Zi-Guang Li. The current hydraulic system simulation technology development status and trends [J]. Machine Tool and Hydraulics, 2003,01:20-22. (in Chinese)

[4] Dong-Sheng Pan, Song-Mao Chen, Hong-Yang Qiu, The present situation and development trend of hydraulic simulation technology [J]. New Technology and Process, 2005,04:7-11. (in Chinese)

[5] De-Seng $\mathrm{Hu}$, Ming Li. Outlook on fluid drive technology in the 21st century [J]. Machine Manufacturing, 2001,05:19-20. (in Chinese)

[6] Ear-Zhuang Yang. Modern transmission technology [J]. Hydraulics, Pneumatics and Seals, 2003. (in Chinese)

[7] MurTenhoff H. Hydraulic Control Technology Development Trend (Part 2). Ji-Guang Yue translation. Engineering Design, 1998 (I): 5-9.

[8] Yi Wang. The combination of Fluid Technology and Electronic Technology and Competition. Chinese Hydraulics and Pneumatics, 1998 (5): 1 - 5 (in Chinese)

[9] Bake. W The Present and future of fluid Power Pore. Einstein. Me. Engrs, 1993,207 (4): 193-212

[10] A-Ning Cheng. The Application of the Hydraulic Simulation Technology and Development [J]. Machine Tool \& Hydraulics, 2004,05:9-10. (in Chinese)

[11] Feng Zhang. The computer simulation technology and its application [J]. Computer Knowledge and Technology (Academic), 2007,19:233+238. (in Chinese) 